
\title{
R Research S Surare \\ Factors associated with hepatic fibrosis and its deterioration among fatty liver elderly: a retrospective cohort study
}

\section{Yu-Shian Lien}

Mackay Medical College

Hsin-Hui Lin

Mackay Memorial Hospital

Chuen-Fei Chen

Mackay Medical College

Meng-Ting Tsou

Mackay Memorial Hospital

Lee-Ching Hwang ( $\nabla$ hlc@mmh.org.tw)

Mackay Memorial Hospital https://orcid.org/0000-0002-7975-5830

Research article

Keywords: elderly, FIB-4 scores, hepatic fibrosis, non-alcoholic fatty liver disease

Posted Date: September 17th, 2020

DOl: https://doi.org/10.21203/rs.3.rs-71535/v1

License: (9) This work is licensed under a Creative Commons Attribution 4.0 International License. Read Full License 


\section{Abstract}

\section{Background}

The progression of hepatic fibrosis in patients with non-alcoholic fatty liver disease (NAFLD) constitutes a major concern in public health as it is associated with many liver-related complications and mortalities such as cirrhosis, varices, or the development of liver cancer. The purpose of this study was to identify predictive factors that deteriorated the degree of hepatic fibrosis, using the FIB-4 score, in elderly NAFLD patients.

\section{Methods}

We collected data from a health examination database. Background information, including demographic characteristics, biochemical blood tests, blood pressures, body mass index (BMI), and abdominal sonography, was collected. We compared the FIB-4 scores from patients' first and last visits at their health check-up, and multiple logistic regression models with $95 \%$ confidence intervals $(\mathrm{Cl})$ were used for adjusting covariates to test the risk factors for deterioration of the FIB-4 scores.

Results:

A total of 742 patients with NAFLD were included in this study, and 534 (72\%) were female. The average age and BMI were $71.8 \pm 5.6$ years and $26.1 \pm 3.0 \mathrm{~kg} / \mathrm{m}^{2}$, respectively. We divided the population based on mild, moderate, and severe hepatic fibrosis groups according to their FIB-4 scores. Patients with severe FIB-4 scores had a baseline total cholesterol and low-density lipoprotein (LDL) lower than the mild and moderate group $(p<0.05)$, and fasting glucose was higher in the mild versus moderate group $(p<0.05)$. After adjusting for covariates, dyslipidemia, including higher serum levels of LDL (odds ratio [OR]:1.02, Cl: 1.00-1.03) and log-triglyceride (OR: 1.68, $\mathrm{Cl}$ : 1.15-2.45), lower levels of log-high-density lipoprotein (OR:1.68, Cl: 1.16-2.42), and longer follow-up years (OR:1.17, Cl: 1.08-1.27) were factors that deteriorated the FIB-4 scores.

\section{Conclusions}

Our study concluded that FIB-4 scores increased over time, and dyslipidemia was a major deteriorating factor for hepatic fibrosis. Therefore, elderly patients with NAFLD should be regularly followed-up and assessed for dyslipidemia to prevent further deterioration of hepatic fibrosis.

\section{Background}

Non-alcoholic fatty liver disease (NAFLD) is characterized by excess accumulation of triglyceride in the hepatocyte and has become the most common cause of liver disease around the world in parallel with the obesity epidemic (1). NAFLD can subsequently progress to cirrhosis, liver failure, and the development of liver cancer (2-4) which places a heavier clinical burden on the healthcare system (5). Compared with the general population, the mortality rate among community-diagnosed NAFLD patients is significantly 
higher, and liver-related death is a leading cause of mortality (2). Therefore, identifying and modifying the risk factors that accelerate the progression of NAFLD is crucial in reducing many liver-related complications and mortalities.

There have been previous studies $(6,7)$ regarding the association between certain risk factors for NAFLD, such as female sex, hypertension, and serum glycated hemoglobin (HbA1c). Elderly patients have a higher risk of liver-related disease and death (2). Based on published literature derived from this systematic review (8), results show that $33.6 \%$ of NAFLD patients may develop fibrosis progression and clinical risk factors included the presence of hypertension, a low aspartate aminotransferase(AST): alanine transaminase(ALT) ratio, and higher steatosis grade. Other studies proposed that the fibrosis stage is an independent risk factor (9), and older age and impaired fasting glucose are associated with liver-related morbidities and mortalities (2).

Despite these clinical findings, studies using a definition of NAFLD liver biopsy have included small numbers of patients. The fibrosis-4 (FIB-4) score is an accepted scoring system which is a simple, inexpensive, and non-invasive method of measuring the extent of hepatic fibrosis in patients with NAFLD [4]. We can screen for patients at risk of advanced hepatic fibrosis and follow-up their long-term prognosis. Further research is needed to assess clinical risk factors associated with the development of progressive fibrosis and strengthen our understanding of disease prevention. The purpose of this study was to identify predictive factors that deteriorated the degree of hepatic fibrosis, using the FIB-4 score, in elderly NAFLD patients.

\section{Materials And Methods}

\section{Study population and methods}

Background information, including demographic characteristics, medical history, personal habit history, blood pressures, body mass index, biochemical blood tests, and abdominal sonography, was collected from an elderly health examination database. Biochemical blood tests included fasting glucose, total protein, albumin, AST, ALT, total cholesterol, low-density lipoprotein (LDL), high-density lipoprotein (HDL), triglyceride (TG), blood urea nitrogen (BUN), uric acid, hemoglobin, platelet count, and creatinine. Each participant underwent an abdominal ultrasound by a well-trained gastrologist to assess the degree of fatty liver disease. Participants who were diagnosed with fatty liver disease by abdominal sonography at baseline and underwent multiple health check-ups were investigated. Individuals younger than 65 yearsold, who had a history of hepatitis $C$ virus infection (or positive anti-HCV), hepatitis B virus infection (or positive $\mathrm{HbsAg}$ ), or regularly consumed alcohol were excluded from this analysis, leaving a database of 742 elderly participants (208 men and 534 women). The FIB-4 index was calculated as AST × age / (platelet count $\times \sqrt{ }$ ALT).

The research was approved by Mackay Memorial Hospital's Institutional Review Board (approval number 18MMHIS137). 


\section{Statistical analysis}

The participants were classified into two groups based on changes of their FIB-4 scores from their first and last regular health exams: the improved or maintained and deteriorated group. SPSS software version 26.0 (IBM Corp.) was used for all statistical analyses. Continuous variables are expressed as means and standard deviations while categorical variables are expressed in the form of frequencies and percentages. Chi-square and Student's t-tests were used to compare characteristics between groups.

Multiple logistic regression models with 95\% confidence intervals $(\mathrm{Cl})$ were used for adjusting covariates to test the risk factors for deterioration of the FIB-4 scores. The odds ratios (ORs) and $95 \%$ confidence intervals (Cls) were presented, and any P-value $<0.05$ was considered statistically significant. Log transformation was used for TG and HDL because their data did not follow a normal distribution.

\section{Results}

A total number of 742 patients were enrolled in the analysis, with a mean age and BMI of $71.8 \pm 5.6$ years and $26.1 \pm 3.0 \mathrm{~kg} / \mathrm{m}^{2}$, respectively. Patients of whom $72.0 \%$ were female, $55.3 \%$ had hypertension, $16.6 \%$ had diabetes mellitus (DM), $25.5 \%$ had hyperlipidemia, and $60.2 \%$ had metabolic syndrome, as shown in table 1. The patient population was divided into 3 groups: mild (20.3\%), moderate $(71.6 \%)$, and severe (8.1\%), based on their FIB-4 scores. We used a lower cutoff point of 1.3 and a higher cutoff point of 2.67 (4). Our analysis revealed that patients with severe FIB-4 scores had a baseline total cholesterol and LDL significantly lower than the mild and moderate group $(p<0.05)$, and fasting glucose was higher in the mild versus moderate group $(p<0.05)$, as shown in table 1 .

After 4.08 years of follow-up, the patients were divided into two groups based on whether or not there was a deterioration of their FIB-4 scores between their first and last visits at their health check-ups. Factors including higher levels of LDL $(p<0.05)$, lower levels of $\mathrm{HDL}(p<0.05)$, and longer follow-up years $(p<$ 0.001 ) showed significant difference between the two groups, as shown in table 2.

In multiple logistic regression models, dyslipidemia, including higher serum levels of $\operatorname{LDL}(O R=1.02, \mathrm{Cl}$ : 1.00-1.03, $\mathrm{p}<0.05)$ and TG (OR = 1.68, Cl: 1.15-2.45, $\mathrm{p}<0.05)$, and lower serum levels of log (HDL) $(\mathrm{OR}=$ 0.43 , Cl: $0.22-0.84, \mathrm{p}<0.014)$ and total cholesterol $(\mathrm{OR}=0.99, \mathrm{Cl}: 0.98-1.00, \mathrm{p}<0.05)$ were associated factors that deteriorated the FIB-4 scores.

In addition, low HDL $(\mathrm{OR}=1.68, \mathrm{Cl}: 1.16-2.42, \mathrm{p}<0.01)$ and high TG $(\mathrm{OR}=1.41, \mathrm{Cl}: 1.02-1.93, \mathrm{p}<0.05)$, along with longer follow-up years $(\mathrm{OR}=1.17, \mathrm{Cl}$ : 1.08-1.27, $\mathrm{p}<0.001)$ were also factors that deteriorated the FIB-4 scores, as shown in table 3.

\section{Discussions}

The principal findings of this study are that FIB-4 scores increased over time, and dyslipidemia was a major deteriorating factor for hepatic fibrosis, as shown in Table 3. Therefore, regardless of whether an 
individual has hepatic steatosis or non-alcoholic steatohepatitis (NASH), all NAFLD patients should be followed-up and assessed for dyslipidemia to prevent further deterioration of hepatic fibrosis.

The impact of chronic liver disease on hepatic lipid metabolism has been mentioned in several studies $(10,11)$. One important finding in this study is that patients with severe NAFLD, as indicated by a FIB-4 score greater than 2.67, were found to have lower lipid levels, specifically total cholesterol and LDL, than patients with moderate and mild FIB-4 scores. The improvement in dyslipidemia in patients with lower FIB-4 scores can possibly be explained by hepatic synthetic failure in patients with cirrhosis $(12,13)$, under the assumption that patients with more severe FIB-4 scores were mostly cirrhotic. However, it is important to note that NAFLD is comprised of a spectrum of conditions ranging from steatosis to NASH, and that the decline in serum total cholesterol, TG, or LDL may indicate that the patient is already precirrhotic or cirrhotic. Furthermore, the amount of decrement in serum total cholesterol, LDL, and HDL have a positive correlation with the severity of liver damage. It is well known that in advanced cirrhosis, the levels of total cholesterol are decreased. In this study, we found that levels of total and LDL cholesterol were both decreased. From a clinically practical standpoint, this could possibly mask the severity of patients' cardiovascular risk due to the improvement in blood lipid tests.

Another interesting finding is that fasting glucose was higher in the mild versus moderate NAFLD group $(p<0.05)$, as shown in Table 1. Although the relationship between the metabolism of glucose and chronic liver disease is not fully understood, there have been several proposed mechanisms for glucose homeostasis in NAFLD (11). A possible explanation for a lower fasting glucose in moderate versus mild NAFLD group is that the liver's ability to mobilize and distribute glucose to peripheral tissues is diminished, this is especially seen in patients with cirrhosis where their liver is severely dysfunctional (14).

Dyslipidemia, characterized by lower levels of HDL, and higher levels of LDL and TG, along with metabolic syndrome, insulin resistance, have long been known to be associated with $\operatorname{NAFLD}(2,15,16)$. However, the exact pathogenesis and progression of non-alcoholic fatty liver still remains to be uncovered. In our study, we found that dyslipidemia was a significant factor that increased the FIB-4 score in patients from their first and last visits. Dyslipidemia is most likely an end result of inflammation and oxidative stress on the liver, which is an oversimplified explanation of the several proposed complex mechanisms and pathways that cause an accumulation of triglycerides in the liver (17). An important metabolic change that plays a part in the pathogenesis of NAFLD is hyperinsulinemia, which increases fatty acid uptake (18), transcriptionally upregulates de novo lipogenesis in the liver (19), and heightens production rates of very-low-density lipoprotein (VLDL) particles (20). These VLDL particles, a major source of circulating TG, may be one of the causes of high TG commonly seen in NAFLD (21). Consequently, the failure of insulin to suppress VLDL secretion leads to dyslipidemia, and overproduction of TG worsens the present steatosis. In essence, dyslipidemia is both a cause and result of NAFLD. Moreover, the increase in VLDL secretion does not compensate for the overproduction of the TG because the free fatty acids incorporated into VLDL particles are from intracellular storage rather than de novo 
synthesis $(20,22)$. These metabolic changes listed above are also discussed on a molecular level $(23)$, but are beyond the scope of our study.

In summary, these mechanisms can all be attributed to insulin resistance (15), which causes a diminished response to insulin in patients with NAFLD. Therefore, it is evident that conditions affiliated with insulin resistance such as obesity and metabolic syndrome are also strongly associated with fatty liver and should be treated along with NAFLD. Given the finding that FIB-4 scores increased over time, we recommend all patients with NAFLD, whether it's simple steatosis or NASH, be regularly followed-up. Although the natural history of NAFLD generally starts from steatosis to NASH, cirrhosis, and even hepatocellular carcinoma (HCC), there have been reports of the development of HCC without apparent cirrhosis (24). More importantly, NAFLD is associated with many hepatic and extrahepatic complications and morbidities $(25,26)$, and this calls for more adequate surveillance of fatty liver patients to prevent further deterioration of their FIB-4 scores.

\section{Strengths And Limitations}

There are several limitations to our study. First, since the progression of fibrosis in patients with NAFLD is affected by many factors such as genetics, the external environment, and intrinsic microbial factors (8, 17), how these factors play a part in the deterioration of hepatic fibrosis in our selected population is unknown. Second, the deterioration rate of the FIB-4 score, which correlates with clinical NAFLD progression, was not conducted in our study, but is crucial in differentiating which groups of patients are at risk of rapid progression that may need appropriate treatment. Third, this study is limited by the overrepresentation of females $(72 \%)$ in the health examination database, which may be seen as a selection bias to our study. However, this may be explained by a previous study that indicated an association between NAFLD and female sex (7). Nevertheless, there is conflicting data about the association of sex and prevalence of NAFLD (27), since the true prevalence of NAFLD is difficult to predict. Fourth, each participant underwent an abdominal ultrasound by a well-trained gastrologist, but not all were able to be conducted by the same gastrologist, which may have different assessments on the degree of fatty liver disease, increasing the possibility of misclassification bias. However, only slight variations may exist from this limitation. Overall, despite these limitations, our study population size was adequate, with 742 participants, and utilized a commonly accepted and non-invasive scoring system (FIB4) to measure the deterioration of hepatic fibrosis, which provides us with generalizability of the results.

\section{Conclusions}

Our study concluded that FIB-4 scores increased over time, and dyslipidemia was a major deteriorating factor for hepatic fibrosis. Patients with more severe hepatic fibrosis, indicated by high FIB-4 scores, might manifest an improvement in their lipid profile - a sign of cirrhotic stage NAFLD - due to hepatic synthetic failure, and should be given proper care and education on prevention of disease progression. Despite patients' severity of NAFD, they should be regularly followed-up to prevent further deterioration of hepatic fibrosis. 


\section{Abbreviations}

AST

aspartate aminotransferase

ALT

alanine aminotransferase

LDL

low-density lipoprotein

HDL

high-density lipoprotein

TG

triglyceride

VLDL

very-low-density lipoprotein

DM

diabetes mellitus

$\mathrm{HCC}$

hepatocellular carcinoma

NAFLD

non-alcoholic fatty liver disease

$\mathrm{NASH}$

non-alcoholic steatohepatitis

NAFL

non-alcoholic fatty liver

FIB-4

fibrosis 4

OR

odds ratio

$\mathrm{Cl}$

confidence interval

\section{Declarations}

\section{Ethics approval and consent to participate}

All authors verify adherence to appropriate ethical standards while conducting this research. The research was approved by Mackay Memorial Hospital's Institutional Review Board (approval number 18MMHIS137).

Consent for publication 
Authors will provide formal written Consent to Publish.

\section{Availability of data and materials}

The datasets generated and/or analyzed during the current study are not publicly available due individual privacy but are available from the corresponding author on reasonable request.

\section{Competing interests}

The authors declare that they have no competing interests.

\section{Funding}

This research received no external funding.

\section{Authors' contributions}

Conceptualization, L-C Hwang; methodology and formal analysis, C-F Chen, Y-S Lien and L-C, Hwang; investigation, M-T Tsou; writing-original draft preparation, Y-S Lien; writing-review and editing, L-C Hwang and $\mathrm{H}-\mathrm{H}$ Lin; project administration, M-T Tsou; All authors contributed to and have approved the final manuscript.

\section{Acknowledgements}

Our thanks to Health Examination Center, MacKay Memorial Hospital for their help in this study.

\section{References}

1. Lonardo A, Sookoian S, Pirola CJ, Targher G. Non-alcoholic fatty liver disease and risk of cardiovascular disease. Metabolism. 2016;65(8):1136-50.

2. Adams LA, Lymp JF, St Sauver J, Sanderson SO, Lindor KD, Feldstein A, et al. The natural history of nonalcoholic fatty liver disease: a population-based cohort study. Gastroenterology. 2005;129(1):113-21.

3. Masuoka HC, Chalasani N. Nonalcoholic fatty liver disease: an emerging threat to obese and diabetic individuals. Ann N Y Acad Sci. 2013;1281:106-22.

4. Shah AG, Lydecker A, Murray K, Tetri BN, Contos MJ, Sanyal AJ. Comparison of noninvasive markers of fibrosis in patients with nonalcoholic fatty liver disease. Clin Gastroenterol Hepatol. 2009;7(10):1104-12.

5. Younossi ZM, Koenig AB, Abdelatif D, Fazel Y, Henry L, Wymer M. Global epidemiology of nonalcoholic fatty liver disease-meta-analytic assessment of prevalence, incidence, and outcomes. 
Hepatology. 2016;64(1):73-84.

6. Ma H, Xu C, Xu L, Yu C, Miao M, Li Y. Independent association of HbA1c and nonalcoholic fatty liver disease in an elderly Chinese population. BMC Gastroenterol. 2013;13(1):3.

7. Pitisuttithum P, Chan WK, Piyachaturawat P, Imajo K, Nakajima A, Seki Y, et al. Predictors of advanced fibrosis in elderly patients with biopsy-confirmed nonalcoholic fatty liver disease: the GOASIA study. BMC Gastroenterol. 2020;20(1):88.

8. Singh S, Allen AM, Wang Z, Prokop LJ, Murad MH, Loomba R. Fibrosis progression in nonalcoholic fatty liver vs nonalcoholic steatohepatitis: a systematic review and meta-analysis of paired-biopsy studies. Clin Gastroenterol Hepatol. 2015;13(4):643 - 54.e9.

9. Angulo P, Kleiner DE, Dam-Larsen S, Adams LA, Bjornsson ES, Charatcharoenwitthaya P, et al. Liver fibrosis, but no other histologic features, is associated with long-term outcomes of patients with nonalcoholic fatty liver disease. Gastroenterology. 2015;149(2):389 - 97.e10.

10. Siddiqui MS, Fuchs M, Idowu MO, Luketic VA, Boyett S, Sargeant C, et al. Severity of nonalcoholic fatty liver disease and progression to cirrhosis are associated with atherogenic lipoprotein profile. Clin Gastroenterol Hepatol. 2015;13(5):1000-8.e3.

11. Chao H-W, Chao S-W, Lin H, Ku H-C, Cheng C-F. Homeostasis of glucose and lipid in non-alcoholic fatty liver disease. Int J Mol Sci. 2019;20(2):298.

12. Ghadir MR, Riahin AA, Havaspour A, Nooranipour M, Habibinejad AA. The relationship between lipid profile and severity of liver damage in cirrhotic patients. Hepat Mon. 2010;10(4):285-8.

13. Juakiem W, Torres DM, Harrison SA. Nutrition in cirrhosis and chronic liver disease. Clin Liver Dis. 2014;18(1):179-90.

14. Majeed A. Hypoglycemia in patients presenting with Liver Cirrhosis. Pak J Med Health Sci. 2017;11(4):1211-3.

15. Gaggini M, Morelli M, Buzzigoli E, Defronzo R, Bugianesi E, Gastaldelli A. Non-alcoholic fatty liver disease (NAFLD) and its connection with insulin resistance, dyslipidemia, atherosclerosis and coronary heart disease. Nutrients. 2013;5(5):1544-60.

16. Niki Katsiki P, Mikhailidis D, Mantzoros S. C. Non-alcoholic fatty liver disease and dyslipidemia: an update. Metabolism. 2016;65:1109-23.

17. Wree A, Broderick L, Canbay A, Hoffman HM, Feldstein AE. From NAFLD to NASH to cirrhosis-new insights into disease mechanisms. Nat Rev Gastroenterol Hepatol. 2013;10(11):627-36.

18. Ferré P, Foufelle F. Hepatic steatosis: a role for de novo lipogenesis and the transcription factor SREBP-1c. Diabetes Obes Metab. 2010;12:83-92.

19. Choi SH, Ginsberg HN. Increased very low density lipoprotein (VLDL) secretion, hepatic steatosis, and insulin resistance. Trends Endocrinol Metab. 2011;22(9):353-63.

20. Kawano Y, Cohen DE. Mechanisms of hepatic triglyceride accumulation in non-alcoholic fatty liver disease. J Gastroenterol. 2013;48(4):434-41. 
21. Fabbrini E, Mohammed BS, Magkos F, Korenblat KM, Patterson BW, Klein S. Alterations in adipose tissue and hepatic lipid kinetics in obese men and women with nonalcoholic fatty liver disease. Gastroenterology. 2008;134(2):424-31.

22. Tessari P, Coracina A, Cosma A, Tiengo A. Hepatic lipid metabolism and non-alcoholic fatty liver disease. Nutr Metab Cardiovasc Dis. 2009;19(4):291-302.

23. Min H-K, Kapoor A, Fuchs M, Mirshahi F, Zhou H, Maher J, et al. Increased hepatic synthesis and dysregulation of cholesterol metabolism is associated with the severity of nonalcoholic fatty liver disease. Cell Metab. 2012;15(5):665-74.

24. Ertle J, Dechêne A, Sowa J-P, Penndorf V, Herzer K, Kaiser G, et al. Non-alcoholic fatty liver disease progresses to hepatocellular carcinoma in the absence of apparent cirrhosis. Int $\mathrm{J}$ Cancer. 2011;128(10):2436-43.

25. Armstrong MJ, Adams LA, Canbay A, Syn W-K. Extrahepatic complications of nonalcoholic fatty liver disease. Hepatology. 2014;59(3):1174-97.

26. Byrne CD, Targher G. NAFLD: a multisystem disease. J Hepatol. 2015;62(1 Suppl):47-64.

27. Sayiner M, Koenig A, Henry L, Younossi ZM. Epidemiology of nonalcoholic fatty liver disease and nonalcoholic steatohepatitis in the United States and the rest of the world. Clin Liver Dis. 2016;20(2):205-14.

\section{Tables}


Table 1 Characteristics of Study Population and Severity Groups of Hepatic Fibrosis According to FIB4 Scores

\begin{tabular}{|c|c|c|c|c|c|}
\hline & \multirow{2}{*}{$\begin{array}{l}\text { Total } \\
\text { subjects }\end{array}$} & \multicolumn{4}{|c|}{ Severity of hepatic fibrosis } \\
\hline & & Mild & Moderate & Severe & \\
\hline Variables & $n=742$ & $\begin{array}{l}n=151 \\
(20.3)\end{array}$ & $\begin{array}{l}n=531 \\
(71.6)\end{array}$ & $\mathrm{n}=60(8.1)$ & $p$ \\
\hline Age (yrs) & $71.8 \pm 5.6$ & $68.9 \pm 4.3$ & $72.2 \pm 5.0$ & $75.2 \pm 6.0$ & $<0.001$ \\
\hline Gender, male (n, \%) & $208(28.0)$ & $27(17.9)$ & $152(28.6)$ & $29(48.3)$ & $<0.001$ \\
\hline Hypertension (n, \%) & $410(55.3)$ & $75(49.7)$ & $298(56.1)$ & $37(61.7)$ & 0.216 \\
\hline Diabetes Mellitus (n, \%) & $123(16.6)$ & $32(21.2)$ & $86(16.2)$ & $5(8.3)$ & 0.070 \\
\hline Hyperlipidemia $(n, \%)$ & $189(25.5)$ & $35(23.2)$ & $142(26.7)$ & $12(20.0)$ & 0.403 \\
\hline Metabolic syndrome (n, \%) & $447(60.2)$ & $94(62.3)$ & $321(60.5)$ & $32(53.3)$ & 0.482 \\
\hline Obese $^{a}(n, \%)$ & $245(33)$ & $52(34.4)$ & $171(32.2)$ & $22(36.7)$ & 0.720 \\
\hline Body mass index $\left(\mathrm{kg} / \mathrm{m}^{2}\right)$ & $26.1 \pm 3.0$ & $25.9 \pm 3.1$ & $26.1 \pm 3.0$ & $26.3 \pm 3.3$ & 0.637 \\
\hline Total cholesterol (mg/dL) & $194.9 \pm 34.1$ & $\begin{array}{l}200.5 \pm \\
37.1\end{array}$ & $\begin{array}{l}194.8 \pm \\
33.1\end{array}$ & $\begin{array}{l}181.6 \pm \\
31.7\end{array}$ & 0.001 \\
\hline LDL (mg/dL) & $116.4 \pm 31.0$ & $\begin{array}{l}122.2 \pm \\
32.7\end{array}$ & $\begin{array}{l}116.1 \pm \\
30.5\end{array}$ & $\begin{array}{l}104.8 \pm \\
28.0\end{array}$ & 0.001 \\
\hline Triglyceride (mg/dL) & $132.5 \pm 66.2$ & $\begin{array}{l}136.1 \pm \\
64.8\end{array}$ & $\begin{array}{l}132.3 \pm \\
67.0\end{array}$ & $\begin{array}{l}125.3 \pm \\
63.4\end{array}$ & 0.558 \\
\hline $\mathrm{HDL}(\mathrm{mg} / \mathrm{dL})$ & $55.0 \pm 13.9$ & $54.6 \pm 13.6$ & $55.2 \pm 13.9$ & $54.2 \pm 15.0$ & 0.807 \\
\hline ALT (IU/L) & $23.8 \pm 13.0$ & $22.6 \pm 10.0$ & $23.2 \pm 12.0$ & $31.8 \pm 22.7$ & $<0.001$ \\
\hline AST (IU/L) & $24.5 \pm 10.0$ & $20.1 \pm 4.2$ & $24.2 \pm 8.0$ & $\begin{array}{l}38.12 \pm \\
20.5\end{array}$ & $<0.001$ \\
\hline Platelet count $\left(10^{3} / \mu \mathrm{L}\right)$ & $218.5 \pm 50.7$ & $\begin{array}{l}227.2 \pm \\
56.7\end{array}$ & $\begin{array}{l}216.4 \pm \\
48.8\end{array}$ & $\begin{array}{l}215.6 \pm \\
50.0\end{array}$ & 0.064 \\
\hline $\begin{array}{l}\text { Fasting plasma glucose } \\
\text { (mg/dL) }\end{array}$ & $110.0 \pm 21.6$ & $\begin{array}{l}114.8 \pm \\
25.6\end{array}$ & $\begin{array}{l}108.5 \pm \\
20.5\end{array}$ & $\begin{array}{l}111.4 \pm \\
18.5\end{array}$ & 0.019 \\
\hline Total protein $(\mathrm{g} / \mathrm{dL})$ & $7.3 \pm 0.4$ & $7.3 \pm 0.3$ & $7.3 \pm 0.4$ & $7.2 \pm 0.5$ & 0.400 \\
\hline Albumin (g/dL) & $4.3 \pm 0.2$ & $4.3 \pm 0.2$ & $4.3 \pm 0.2$ & $4.3 \pm 0.3$ & 0.184 \\
\hline
\end{tabular}

Note. LDL: low-density lipoprotein, HDL: high-density lipoprotein, AST: aspartate aminotransferase, ALT: alanine aminotransferase; ${ }^{a}$ Obese: body mass index $>27\left(\mathrm{~kg} / \mathrm{m}^{2}\right)$. 
Table 2 Factors Associated with Deterioration of FIB4_Score in NAFLD Elderly

\begin{tabular}{|llll|}
\hline & No deterioration & Deterioration & \\
\hline Variables & $\mathrm{n}=248$ & $\mathrm{n}=494$ & $p$ \\
\hline Age (yrs) & $72.0 \pm 5.5$ & $71.7 \pm 5.1$ & 0.448 \\
\hline Gender (female, $\mathrm{n}, \%)$ & $170(68.5)$ & $364(73.7)$ & 0.142 \\
\hline Hypertension (n, \%) & $139.0(56.0)$ & $271.0(54.9)$ & 0.758 \\
\hline Diabetes mellitus (n, \%) & $43.0(17.3)$ & $80.0(16.2)$ & 0.693 \\
\hline Hyperlipidemia (n, \%) & $61.0(24.6)$ & $128.0(25.9)$ & 0.698 \\
\hline Metabolic syndrome (n, \%) & $146(58.9)$ & $301(60.9)$ & 0.589 \\
\hline Obese ${ }^{\text {a }(\mathrm{n}, \%)}$ & $81(32.7)$ & $164(33.2)$ & 0.883 \\
\hline Total cholesterol (mg/dL) & $192.7 \pm 33.8$ & $196.0 \pm 34.2$ & 0.215 \\
\hline LDL (mg/dL) & $113.2 \pm 31.1$ & $118.0 \pm 30.9$ & 0.045 \\
\hline Triglyceride (mg/dL) & $126.0 \pm 65.7$ & $135.8 \pm 66.3$ & 0.060 \\
\hline High triglyceride ${ }^{\mathrm{b}}$ & $100(40.3)$ & $234(47.4)$ & 0.069 \\
\hline HDL (mg/dL) & $56.4 \pm 14.2$ & $54.2 \pm 13.7$ & 0.042 \\
\hline Low HDL ${ }^{\mathrm{c}}$ & $52(21.0)$ & $154(31.2)$ & 0.003 \\
\hline Fasting plasma glucose (mg/dL) & $110.8 \pm 21.0$ & $109.7 \pm 22.0$ & 0.518 \\
\hline Total protein (g/dL) & $7.3 \pm 0.4$ & $7.3 \pm 0.4$ & 0.149 \\
\hline Albumin (g/dL) & $4.3 \pm 0.2$ & $4.3 \pm 0.3$ & 0.593 \\
\hline AST (IU/L) & $27.2 \pm 13.3$ & $23.1 \pm 7.6$ & $<0.001$ \\
\hline ALT (IU/L) & $25.2 \pm 16.1$ & $23.0 \pm 11.1$ & 0.060 \\
\hline Follow-up years (yrs) & $3.6 \pm 2.0$ & $4.3 \pm 2.3$ & $<0.001$ \\
\hline
\end{tabular}

Note. LDL: low-density lipoprotein, HDL: high-density lipoprotein, AST: aspartate aminotransferase, ALT: alanine aminotransferase; ${ }^{\text {a }}$ Obese: body mass index $>27\left(\mathrm{~kg} / \mathrm{m}^{2}\right) ;{ }^{\mathrm{b}}$ High triglyceride is $<150 \mathrm{mg} / \mathrm{dL}$; ${ }^{\mathrm{c}}$ Low $\mathrm{HDL}$ is $<50 \mathrm{mg} / \mathrm{dL}$ in females, $<40 \mathrm{mg} / \mathrm{dL}$ in males.

Table 3 Adjusted Odds Ratios of Dyslipidemia for Deterioration of FIB-4 scores 


\begin{tabular}{|c|c|c|c|c|c|}
\hline & & \multirow[t]{2}{*}{ Adjusted Odds Ratios } & \multicolumn{3}{|c|}{$95 \% \mathrm{Cl}$} \\
\hline & & & $U L$ & $L L$ & $p$ \\
\hline \multirow[t]{4}{*}{ Model 1} & LDL (mg/dL) & 1.02 & 1.00 & 1.03 & 0.016 \\
\hline & Total cholesterol $(\mathrm{mg} / \mathrm{dL})$ & 0.99 & 0.98 & 1.00 & 0.045 \\
\hline & Log (triglyceride)* & 1.68 & 1.15 & 2.45 & 0.008 \\
\hline & Follow-up years (yrs) & 1.17 & 1.08 & 1.27 & $<0.001$ \\
\hline Model 2 & $\log (H D L)^{\star}$ & 0.43 & 0.22 & 0.84 & 0.014 \\
\hline Model 3 & Low HDL ${ }^{a}$ & 1.68 & 1.16 & 2.42 & 0.006 \\
\hline Model 4 & High triglyceride ${ }^{b}$ & 1.41 & 1.02 & 1.93 & 0.037 \\
\hline
\end{tabular}

Note. Models were adjusted with age, sex, body mass index, follow-up years, diabetes, and hypertension. Cl: confidence interval, LL: lower limit, UL: upper limit, LDL: low-density lipoprotein, HDL: high-density lipoprotein; ${ }^{a}$ Low HDL is $<50 \mathrm{mg} / \mathrm{dL}$ in females, $<40 \mathrm{mg} / \mathrm{dL}$ in males. ${ }^{\mathrm{b}}$ High triglyceride is $>150 \mathrm{mg} / \mathrm{dL}$. *Non-normal distribution parameters were log transformed for analysis. 